\title{
Downregulation of ubiquitin-associated protein 2-like with a short hairpin RNA inhibits human glioma cell growth in vitro
}

\author{
BING ZHAO $^{1,2}$, GANG ZONG ${ }^{1,2}$, YONGSHENG XIE ${ }^{1,2}$, JIA LI ${ }^{1,2}$, HONGLIANG WANG ${ }^{1,2}$ and ERBAO BIAN ${ }^{1,2}$ \\ ${ }^{1}$ Department of Neurosurgery, The Second Affiliated Hospital of Anhui Medical University; \\ ${ }^{2}$ Cerebral Vascular Disease Research Center, Anhui Medical University, Hefei, Anhui 230601, P.R. China
}

Received December 12, 2014; Accepted August 10, 2015

DOI: $10.3892 /$ ijmm.2015.2323

\begin{abstract}
Ubiquitin-associated protein 2-like (UBAP2L), which contains a ubiquitin-associated (UBA) domain near its $\mathrm{N}$-terminus, has been indicated in the pathogenesis of several human cancers, including multiple myeloma, hepatocellular carcinoma and malignant ovarian tumors. However, the role of UBAP2L in human glioma remains unknown. In the present study, UBAP2L was widely expressed in multiple glioma cell lines. To further examine the effects of UBAP2L on glioma growth, lentivirus-mediated short hairpin RNA (shRNA) was employed to knockdown UBAP2L expression in the glioblastoma cell lines. Depletion of UBAP2L significantly inhibited the proliferation and colony formation ability, as determined by MTT and colony formation assays. Cell cycle analysis showed that UBAP2L knockdown induced $G_{0} / G_{1}$ phase arrest in U251 and U373 cells, while S phase arrest was induced in A172 cells. These results suggest that UBAP2L has a key role in glioma cell growth, and may act as an oncogene to promote malignant glioma development.
\end{abstract}

\section{Introduction}

Glioma is the most common type of primary malignant brain tumors in adults, accounting for $44.69 \%$ of intracranial tumors. Despite intense therapy incorporating surgical resection, radiation therapy and chemotherapy, the prognosis for glioblastoma patients remains poor (1). Glioma is a genetic disease that is caused by multiple oncogenic signaling pathways and mutant genes (2). In glioma cells, aberrant oncogenic factors cause activation of the signaling pathways that are essential for cell proliferation and survival. Therefore,

Correspondence to: Dr Bing Zhao, Department of Neurosurgery, The Second Affiliated Hospital of Anhui Medical University, Anhui Medical University, 678 Fu Rong Road, Hefei, Anhui 230601, P.R. China

E-mail: bingzhaodr@163.com

Key words: ubiquitin-associated protein 2-like, glioma, short hairpin RNA, proliferation, cell cycle selective degradation of such oncogenic factors may be a promising approach to control glioma tumorigenesis and progression.

One of the major protein degradation pathways is the ubiquitin proteasome system (UPS) (3-7), which is known as a cellular tool for selective degradation of target proteins and has been shown to have an important role in multiple physiological processes, such as signaling regulation, transcriptional regulation, cell survival, migration, apoptosis and DNA damage response (4,7). The UPS is composed of a 76-amino acid protein ubiquitin (Ub), a multi-subunit protein organelle $26 \mathrm{~S}$ proteasome, and a three-step enzymatic cascade of Ub-activating (E1), Ub-conjugating (E2) and Ub-ligase (E3) enzymes (6), which are essential for the consistent recycling of a plethora of proteins with distinct structural and functional roles within the cell, including cell cycle regulation (3). A previous study has confirmed the involvement of UPS in regulation of the cell cycle in malignant gliomas (6).

The ubiquitin-associated protein 2-like (UBAP2L) gene is located at human chromosome 1q21.3 and is involved in the pathogenesis of several types of human cancer (8), including multiple myeloma (9), hepatocellular carcinoma (HCC) (10) and malignant ovarian tumors (11). Additionally, UBAP2L contains a UBA domain near its $\mathrm{N}$-terminus, which is a recurring sequence of $\sim 45$ amino acids and is associated with the UPS, DNA excision repair and cell signaling via protein kinases (12). Thus, UBAP2L may have a vital role in regulation of cell cycle and apoptosis by utilizing the UPS. However, the function of UBAP2L in glioma has not been investigated.

To study the potential role of UBAP2L in human glioma, loss of function analysis using lentivirus-mediated short hairpin RNA (shRNA) was performed in glioblastoma cell lines. Subsequently, the effects of UBAP2L silencing were investigated on cell proliferation, colony formation and cell cycle progression.

\section{Materials and methods}

Cell culture. Human glioma U251, U87MG, U373, A172 and U-118MG cell lines, as well as the human embryonic kidney (HEK) 293T cell line, were purchased from the Cell Bank of Type Culture Collection of Chinese Academy of Sciences (Shanghai, China). All the cell lines were maintained in Dulbecco's modified Eagle's medium (HyClone, Logan, UT, 
USA) supplemented with $10 \%$ fetal bovine serum (HyClone) at $37^{\circ} \mathrm{C}$ in a humidified atmosphere of $5 \% \mathrm{CO}_{2}$.

Construction of UBAP2L shRNA lentivirus and cell infection. The shRNA (5'-GCCAATACTGATGATAACTATCTCGAG ATAGTTATCATCAGTATTGGCTTTTT-3')-targeting human UBAP2L gene (NM_001127320.1) was synthesized, annealed and ligated into the green fluorescent protein (GFP)-encoding pFH-L vector (Shanghai Hollybio Co., Ltd., Shanghai, China). ScrambledshRNA(5'-CTAGCCCGGTTCTCCGAACGTGTC ACGTATCTCGAGATACGTGACACGTTCGGAGAATTT TTTTAAT- $3^{\prime}$ ) was used as the control. The lentiviral-based shRNA-expressing vector was verified by DNA sequencing. The generated plasmid was termed pFH-L-shUBAP2L or -shCon. To avoid the possible off-target effect, another shRNA (5'-CGCAGCAGAATACCTTTCATCTCGAGATG AAAGGGTATTCTGCTGCGTTTTT-3') was used to obtain comparable results. Recombinant lentiviral vectors and packaging vectors ( $\mathrm{pVSVG}-\mathrm{I}$ and $\mathrm{pCMV} \Delta \mathrm{R} 8.92$ ) were subsequently transfected into 293T cells using Lipofectamine 2000 (Invitrogen Life Technologies, Carlsbad, CA, USA) according to the manufacturer's instructions. Supernatants containing lentiviruses expressing UBAP2L shRNA or control shRNA were harvested 3 days after transfection. Subsequently, the lentiviruses were purified by ultracentrifugation, and the titer of lentiviruses was determined as described previously (13). U251 and U373 cells were infected with the lentivirus constructs at a multiplicity of infection of 10 and uninfected cells were used as negative controls. The infection efficiency was determined by counting GFP-expressing cells under an Olympus BX50 Brightfield/Fluorescence microscope (Olympus Corp., Tokyo, Japan) $96 \mathrm{~h}$ after infection.

Reverse transcription-polymerase chain reaction (RT-PCR). Total RNA was extracted from the cultured cells using TRIzol reagent (Invitrogen Life Technologies). cDNA was synthesized using M-MLV Reverse Transcriptase (Promega Corp., Madison, WI, USA) according to the manufacturer's instructions. In brief, a mixture including $1.5 \mu \mathrm{g}$ of total RNA, $0.75 \mu \mathrm{g}$ oligo(dT) primer (Sangon Biotech Co., Ltd., Shanghai, China) and nuclease-free water was heated at $70^{\circ} \mathrm{C}$ for $5 \mathrm{~min}$ and subsequently cooled on ice for a further $5 \mathrm{~min}$. In addition, $4 \mu \mathrm{l}$ M-MLV buffer, $1.25 \mu \mathrm{l}$ dNTP, $0.5 \mu 1$ RNasin and $0.75 \mu \mathrm{l}$ M-MLV-RT were supplemented to the mixture up to a final volume of $20 \mu \mathrm{l}$, followed by incubation at $42^{\circ} \mathrm{C}$ for $60 \mathrm{~min}$.

$R T$-quantitative PCR (RT-qPCR). RT-qPCR analysis was performed using SYBR-Green Master mix kits (Takara Bio, Inc., Tokyo, Japan) according to the manufacturer's instructions for the Bio-Rad Connect Real-Time PCR platform (Bio-Rad, Hercules, CA, USA). Briefly, each PCR reaction mixture, containing $10 \mu \mathrm{l}$ of $2 \mathrm{X}$ SYBR-Green Master mix, $0.8 \mu \mathrm{l}$ of sense and antisense primers $(2.5 \mu \mathrm{M})$ and $5 \mu \mathrm{l}$ of cDNA (10 ng), was run for 40 cycles with an initial denaturation at $95^{\circ} \mathrm{C}$ for $60 \mathrm{sec}$, and subsequent denaturation at $95^{\circ} \mathrm{C}$ for $5 \mathrm{sec}$, and annealing and extension at $60^{\circ} \mathrm{C}$ for $30 \mathrm{sec}$. The relative quantification of gene expression was calculated using the $2^{-\Delta \Delta C T}$ method (14). The primer sequences for PCR amplification of the UBAP2L gene were forward, 5'-ACACAATCCCCATCACTGGT-3' and reverse, 5'-CAGAGGAGAAGACGGAGGTG-3'. $\beta$-actin was used as an internal control. The primer sequences of $\beta$-actin were forward, 5'-GTGGACATCCGCAAAGAC-3' and reverse, 5'-AAAGGGTGTAACGCAACTA-3'.

Western blot analysis. U251 and U373 cells were respectively collected 5 days after infection and total proteins were isolated from the cells and quantitated by the bicinchoninic acid method. Proteins were separated by SDS-PAGE and transferred onto polyvinylidene difluoride membrane (Millipore, Billerica, MA, USA). Proteins were probed overnight at $4^{\circ} \mathrm{C}$ with the rabbit anti-UBAP2L (1:2,000 dilution; cat. no. ab70319; Abcam, Cambridge, UK) or rabbit anti-GAPDH (1:50,000 dilution; cat. no. 10494-1-AP; Proteintech Group, Inc., Chicago, IL, USA) primary antibodies, followed by incubation with horseradish peroxidase-conjugated goat anti-rabbit immunoglobulin $\mathrm{G}$ (1:5,000 dilution; cat. no. sc-2054; Santa Cruz Biotechnology, Inc., Dallas, TX, USA) at room temperature for $2 \mathrm{~h}$. GAPDH was used as the internal standard. Subsequently, proteins were detected by the respective antibodies according to the manufacturer's instructions for the ECL kit (Pierce Biotechnology, Inc., Rockford, IL, USA) and exposed to X-ray films that were quantified using the Amersham Image Scanner with LabScan ImageQuant TL Software (Amersham Pharmacia Biotech, Piscataway, NJ, USA).

MTT assay. After 4 days of lentivirus infection, U251 and U373 cells were trypsinized, resuspended and seeded in 96-well plates at a concentration of 2,000 and 2,500 cells/well, respectively. On the following day, the cultured cells were incubated with $1,2,3,4,5$ and $10 \mu 1$ of $5 \mathrm{mg} / \mathrm{ml}$ MTT (Sigma-Aldrich, St. Louis, MO, USA), and incubation was continued for $3 \mathrm{~h}$. Following the careful removal of the remaining medium, $100 \mu \mathrm{l}$ of acidified isopropanol was added to each well at the end of the incubation. The absorbance was measured at $595 \mathrm{~nm}$ on the spectrophotometer.

Colony formation assay. After 4 days of lentivirus infection, U251 and U373 cells were incubated in 6-well plates at a concentration of 200 and 400 cells/well, respectively. The medium was changed at regular time intervals. After 11 days of cultivation at $37^{\circ} \mathrm{C}$, the natural colonies were washed by phosphate-buffered saline (PBS) and fixed with $4 \%$ paraformaldehyde (Sigma-Aldrich) for $30 \mathrm{~min}$ at room temperature. The colonies were stained using crystal violet (Sigma-Aldrich) for $10 \mathrm{~min}$, were subsequently washed with water and were air-dried. The total number of colonies including $>50$ cells was counted under fluorescence microscopy.

Fluorescence-activated cell sorting analysis. The cell cycle distribution was analyzed using flow cytometry with propidium iodide (PI) staining. Briefly, lentivirus-transduced cells ( $3 \times 10^{5}$ cells/dish) were seeded in $6-\mathrm{cm}$ dishes and incubated at $37^{\circ} \mathrm{C}$ for $40 \mathrm{~h}$. Cells were harvested following trypsinization, washed with PBS and fixed in 70\% cold ethanol overnight at $4^{\circ} \mathrm{C}$. Cells were subsequently collected by centrifugation and resuspended in PBS containing $100 \mu \mathrm{g} / \mathrm{ml}$ of DNase-free RNase and $50 \mu \mathrm{g} / \mathrm{ml}$ PI (Sigma-Aldrich), and incubated in the dark at room temperature for $1 \mathrm{~h}$. Finally, the suspension was subjected to FACSCalibur flow cytometer analysis (BD Biosciences, San Jose, CA, USA). The fractions of the cells in 
the $\mathrm{G}_{0} / \mathrm{G}_{1}, \mathrm{~S}$ and $\mathrm{G}_{2} / \mathrm{M}$ phases were analyzed with the ModFit LT DNA analysis program (Verity Software House, Topsham, ME, USA).

Statistical analysis. All the data are expressed as the mean \pm standard deviation of at least triplicate determination. The Student's t-test was used to evaluate the differences between groups using the SPSS 13.0 software (SPSS, Inc., Chicago, IL, USA). $\mathrm{P}<0.05$ was considered to indicate a statistically significant difference.

\section{Results}

Lentivirus-mediated shRNA inhibits UBAP2L $m R N A$ and protein expression in U251 and U373 cells. The expression of UBAP2L mRNA was first detected in five glioma cell lines, U251, U87MG, A172, U373 and U-118MG, using RT-qPCR. As shown in Fig. 1A, UBAP2L mRNA was widely expressed in all five cell lines. Subsequently, U251, U373 and A172 cell lines were applied for the loss-of-function investigation in the following study. A lentiviral vector system was constructed to express shRNA targeting of UBAP2L with a GFP reporter gene. To determine whether the recombinant lentiviruses could infect glioma cell lines, cells infected with Lv-shUBAP2L and Lv-shCon were observed under light and fluorescence microscopes. Four days post-infection, $>90 \%$ of U251 and U373 cells expressed GFP (Fig. 1B), suggesting high-efficiency infection by the lentivirus. There was no significant difference concerning cell morphology and cell growth between the Lv-shCon and Con groups. To verify the knockdown efficiency, the transcription and translation levels of UBAP2L in U251 and U373 cells were assessed by RT-qPCR and western blot analysis following 7 days of lentivirus infection. Compared with the Lv-shCon groups, the mRNA levels of UBAP2L in the Lv-shUBAP2L groups were markedly reduced by $61.0 \%$ in U251 cells and $88.9 \%$ in U373 cells (Fig. 1C and D). The protein levels of UBAP2L were concomitantly decreased in U251 and U373 cells (Fig. 1E and F). These results indicated that the constructed lentiviruses could successfully knockdown UBAP2L expression in glioma cells.

Effects of UBAP2L knockdown on proliferation of U251 and U373 cells. To investigate the effect of UBAP2L knockdown on cell proliferation, the MTT assay was performed in U251 and U373 cells following 7 days of lentivirus infection. In comparison with Lv-shCon groups, depletion of UBAP2L significantly inhibited the proliferation of glioma cells $(\mathrm{P}<0.001)$ by a $19.8 \%$ reduction in $\mathrm{U} 251$ cells (Fig. $2 \mathrm{~A})$ and $46.5 \%$ reduction in U373 cells (Fig. 2B). These results suggested that UBAP2L accelerated glioma cell proliferation.

Effects of UBAP2L knockdown on colony formation of U251 and U373 cells. To detect whether UBAP2L has an influence on the colony-forming capacity of glioma cells, the colony formation assay was performed in U251 and U373 cells. The size of the single colony and the number of colonies formed in the Lv-shUBAP2L groups were significantly decreased when compared with the Lv-shCon and Con groups $\left[\left(\mathrm{P}_{\mathrm{U} 251}<0.01\right.\right.$; Fig. 3A and $\mathrm{C})$; $\left(\mathrm{P}_{\mathrm{U} 373}<0.001\right.$; Fig. 3B and $\left.\mathrm{D}\right)$ ], suggesting that
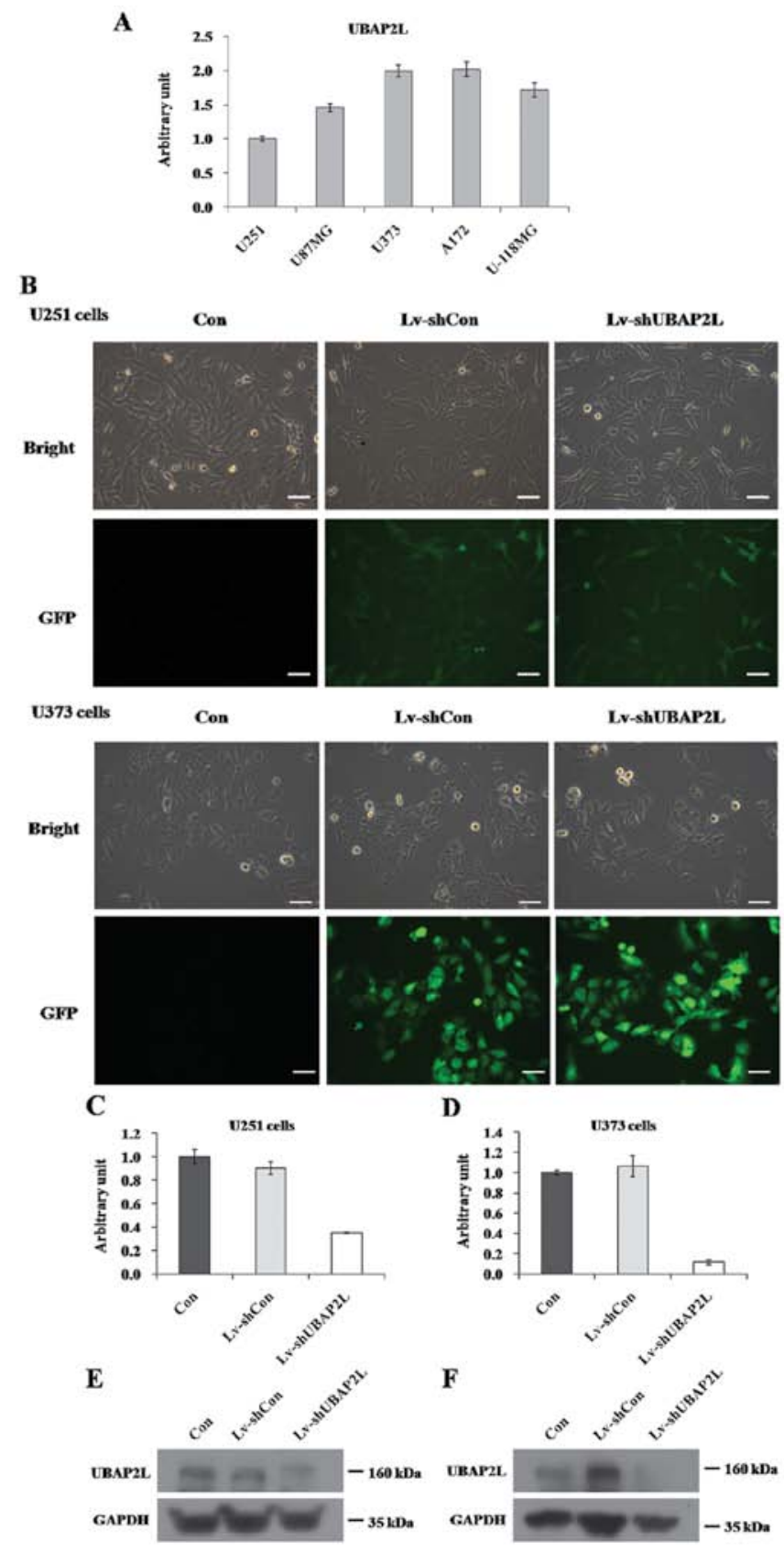

Figure 1. Knockdown of ubiquitin-associated protein 2-like (UBAP2L) by lentivirus-mediated short hairpin RNA (shRNA) in glioma cells. (A) Reverse transcription-quantitative polymerase chain reaction (RT-qPCR) analysis of UBAP2L expression in U251, U87MG, A172, U373 and U-118MG glioma cells. (B) Determination of infection efficiency in human glioma cells. Representative images of U251 and U373 cells after 4 days of lentivirus infection are shown. (C and D) Expression analysis of UBAP2L mRNA and ( $E$ and F) protein levels in U251 and U373 cells by RT-qPCR and western blot analysis. The $\beta$-actin gene and the GAPDH protein were the internal controls for RT-qPCR and western blot analysis, respectively. Con, cells without infection; $\mathrm{Lv}$-shCon, cells infected with the Lv-shCon; Lv-shUBAP2L, cells infected with the Lv-shUBAP2L. Scale bar, $100 \mu \mathrm{m}$.

the reduced expression of UBAP2L could significantly inhibit colony formation in glioma cells.

Effects of UBAP2L knockdown on cell cycle distribution of U251 and U373 cells. To elucidate the mechanism underlying cell proliferation inhibition, the cell cycle distribution of all three groups was analyzed by flow cytometry in U251 and U373 cells 
$\mathbf{A}$

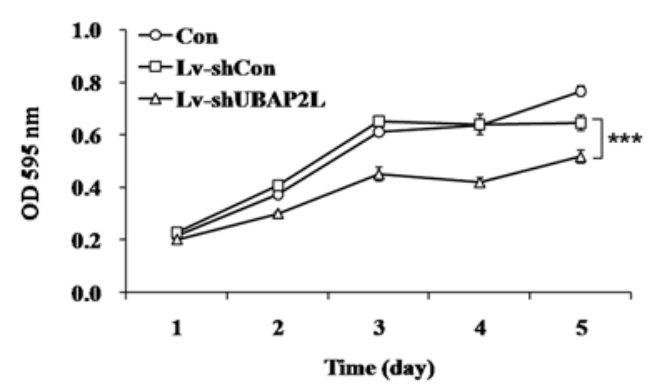

B

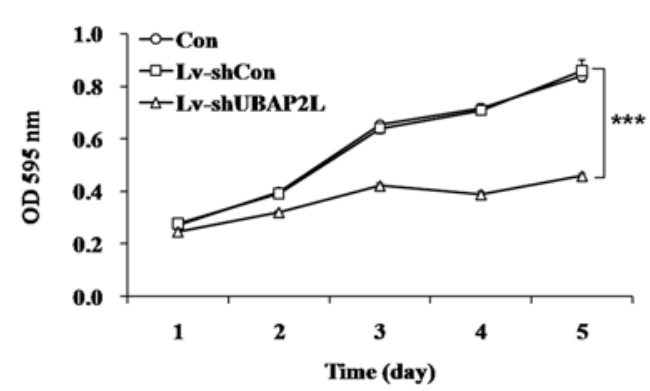

Figure 2. Effect of ubiquitin-associated protein 2-like (UBAP2L) knockdown on the proliferation of (A) U251 and (B) U373 glioblastoma cells. Cell proliferation in Lv-shUBAP2L groups was significantly inhibited, as detected by the MTT assay. Con, cells without infection; Lv-shCon, cells infected with the Lv-shCon; Lv-shUBAP2L, cells infected with the Lv-shUBAP2L. ${ }^{* * *} \mathrm{P}<0.001$.

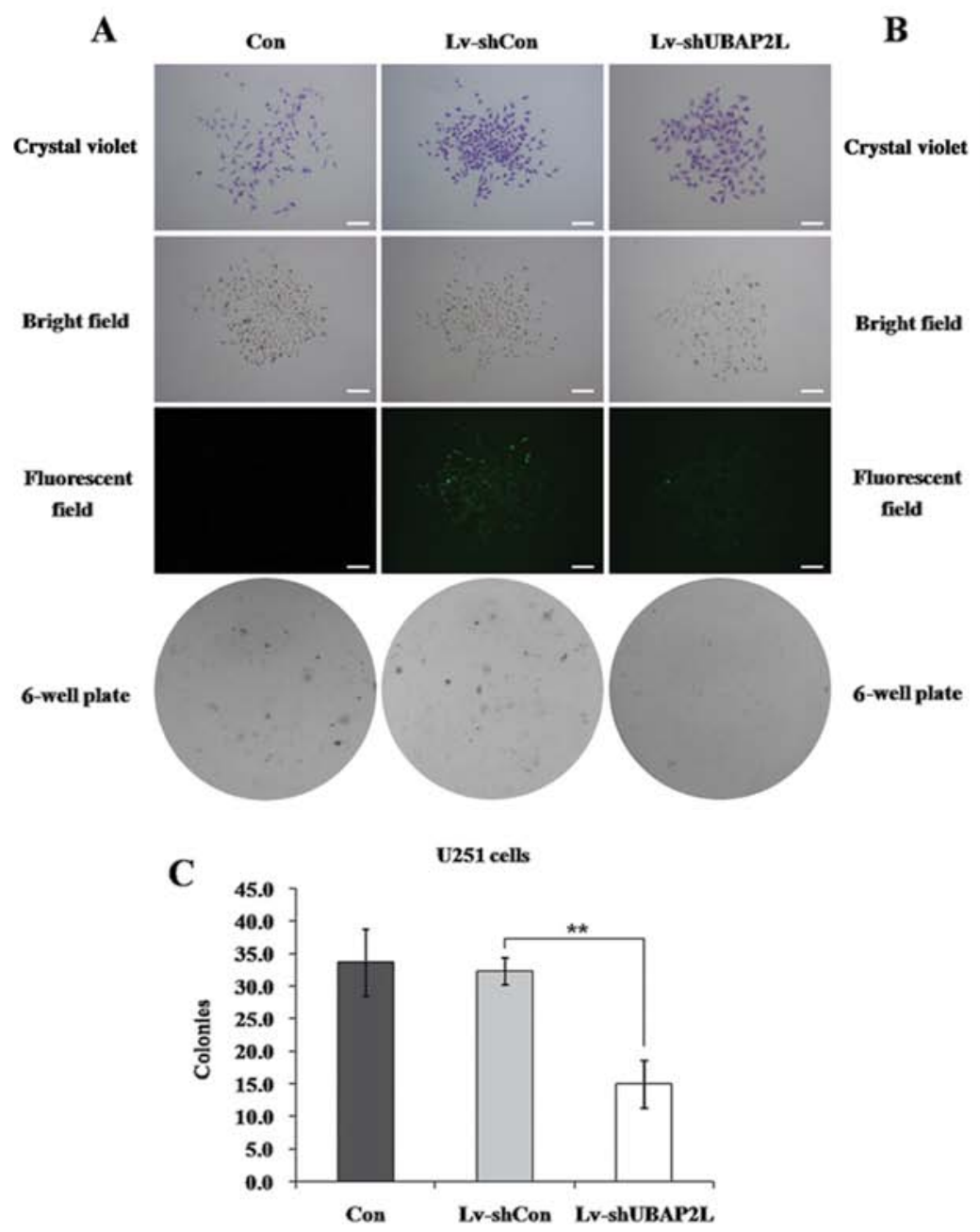

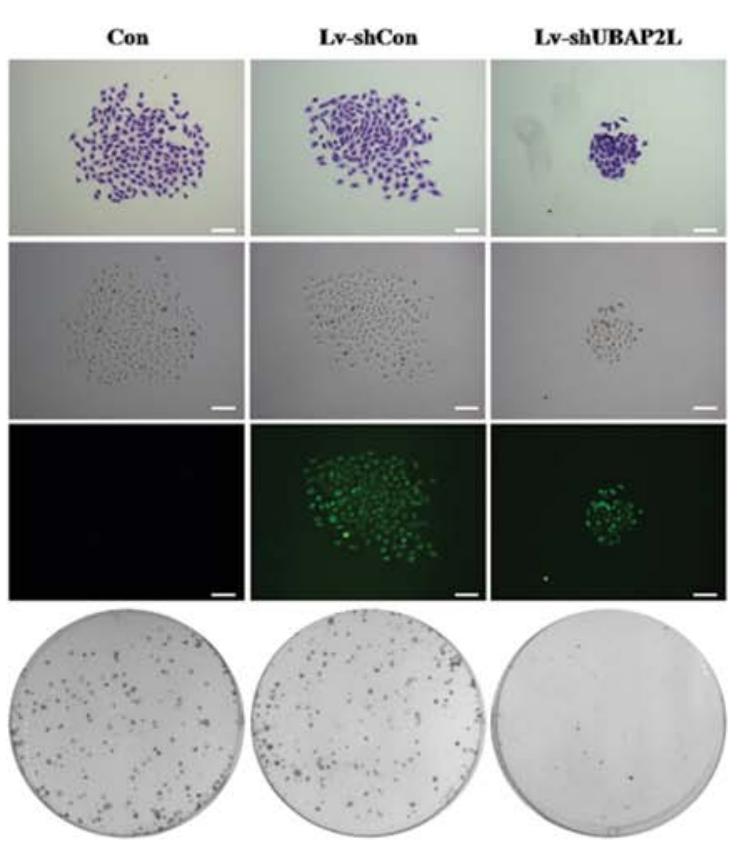

D

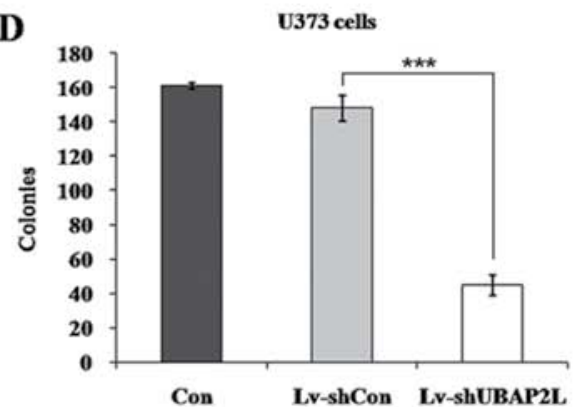

Figure 3. Knockdown of ubiquitin-associated protein 2-like (UBAP2L) inhibited the colony-forming ability of U251 and U373 cells. (A and B) Representative images of colonies under light and fluorescence microscope. (C and D) Statistical analysis of the number of colonies with crystal violet staining. Con, cells without infection; Lv-shCon, cells infected with the Lv-shCon; Lv-shUBAP2L, cells infected with the Lv-shUBAP2L. Scale bar, $250 \mu$ m. ${ }^{* *} \mathrm{P}<0.01,{ }^{* * *} \mathrm{P}<0.001$.

following lentivirus infection (Fig. 4A and B). Compared to the

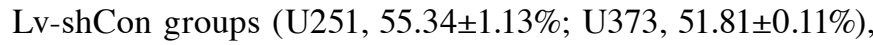
the cell percentages of $\mathrm{G}_{0} / \mathrm{G}_{1}$ phase in the Lv-shUBAP2L

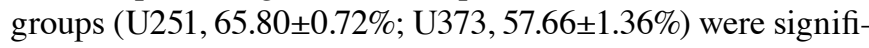
cantly increased in the U251 and U373 cells (Fig. 4C and D). There was no evident difference between the Lv-shCon and Con groups in all the cell lines. These results indicated that knockdown of UBAP2L could inhibit glioma cell growth via blockade of cell cycle progression.

Effects of UBAP2L knockdown on proliferation and cell cycle distribution of A172 cells. Furthermore, the effects of UBAP2L knockdown on cell growth were confirmed in A172 cells. More than $80 \%$ of A172 cells expressed GFP following 

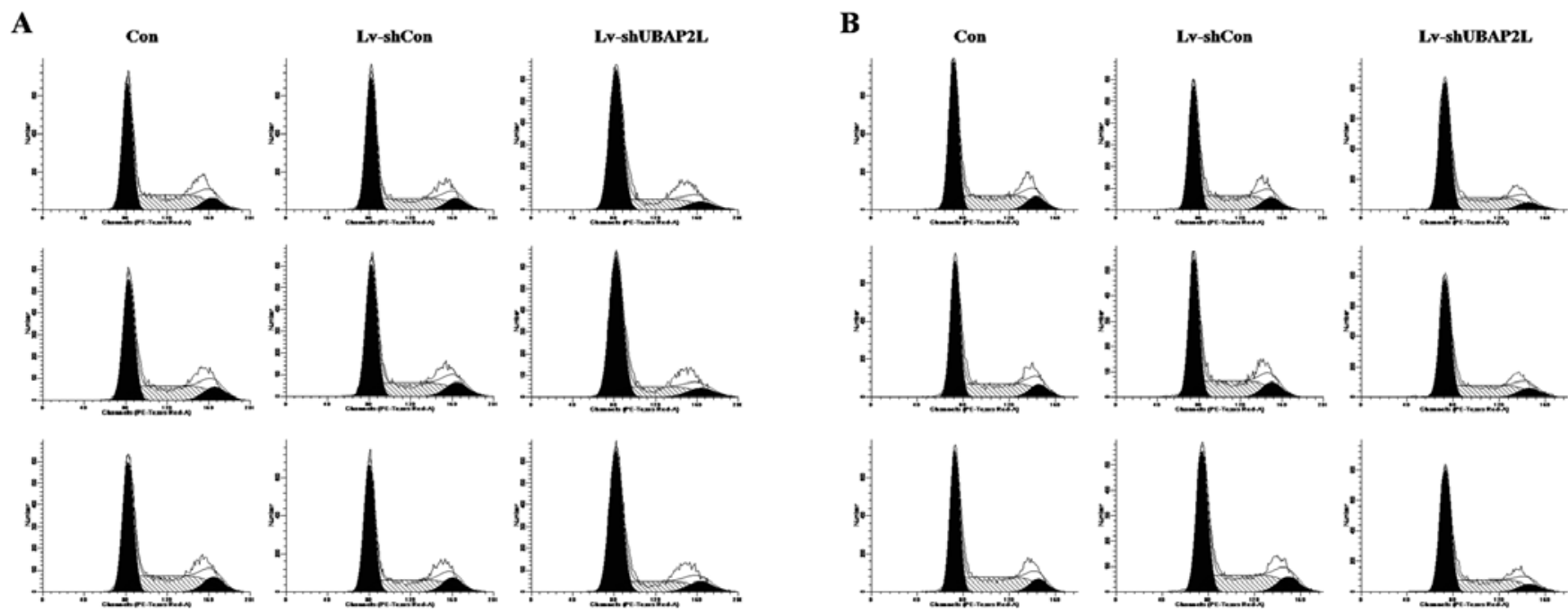

C U251 cells

D U373 cells
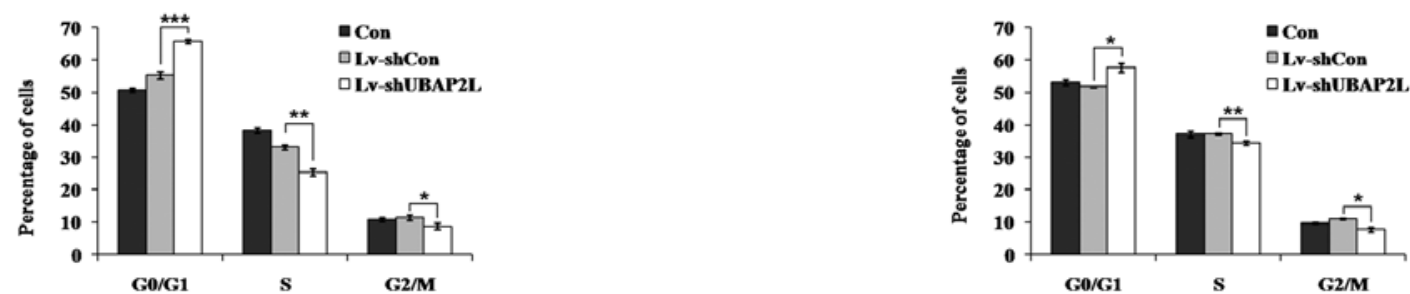

Figure 4. Knockdown of ubiquitin-associated protein 2-like (UBAP2L) induced cell cycle arrest at $\mathrm{G}_{0} / \mathrm{G}_{1}$ phase in U251 and U373 cells. (A and B) Cell cycle distribution of U251 and U373 cells was analyzed by flow cytometry. (C and D) The cell population of $\mathrm{G}_{0} / \mathrm{G}_{1}$ phases was increased in Lv-shUBAP2L groups. Con, cells without infection; Lv-shCon, cells infected with the Lv-shCon; Lv-shUBAP2L, cells infected with the Lv-shUBAP2L. ${ }^{*} \mathrm{P}<0.05,{ }^{* *} \mathrm{P}<0.01,{ }^{* * * *} \mathrm{P}<0.001$.
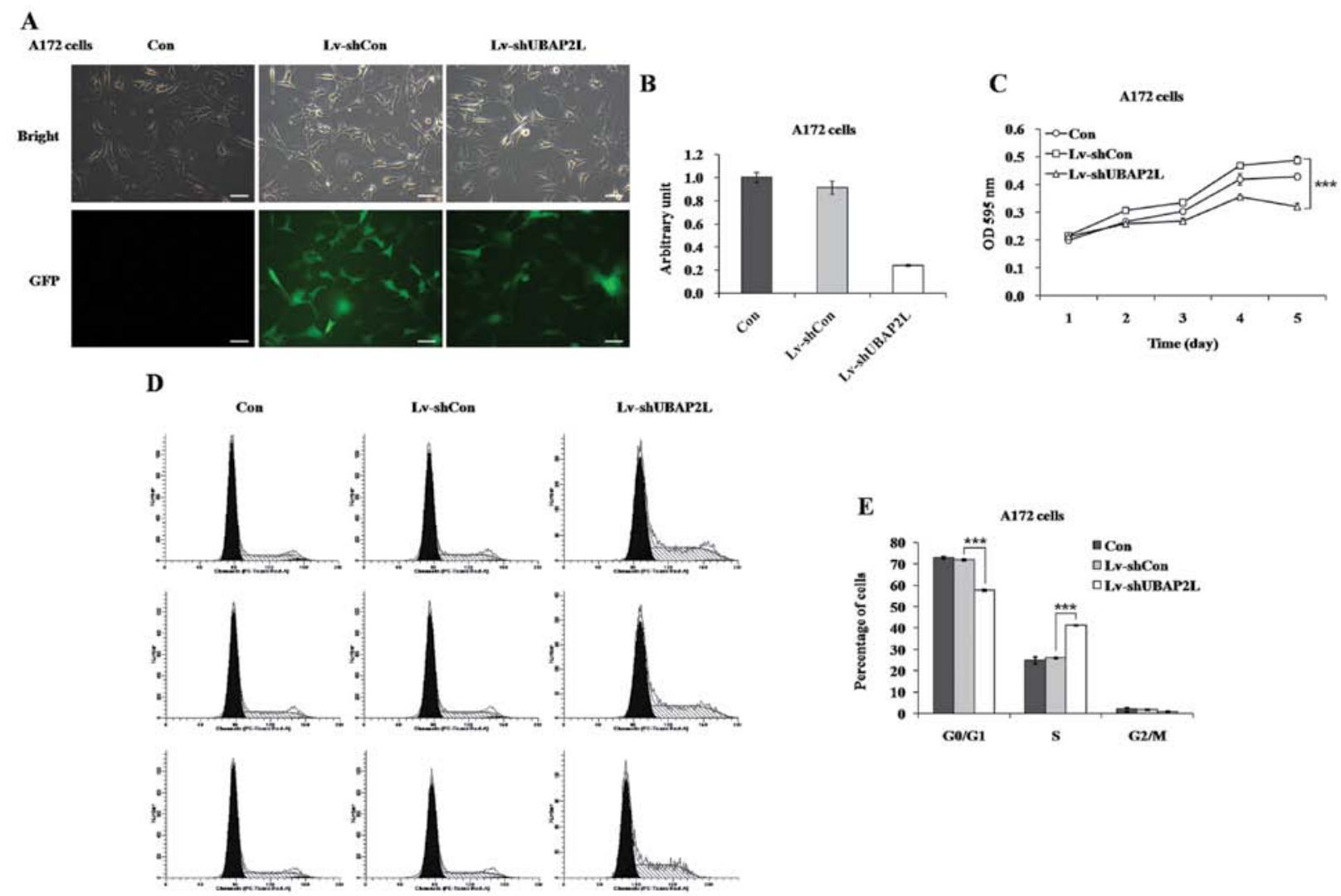

Figure 5. Function analysis of ubiquitin-associated protein 2-like (UBAP2L) by short hairpin RNA (shRNA) in A172 cells. (A) Representative images of A172 cells after 4 days of lentivirus infection are shown. (B) Reverse transcription-quantitative polymerase chain reaction analysis of UBAP2L mRNA levels in A172 cells. $\beta$-actin was the internal control. (C) Cell proliferation in Lv-shUBAP2L groups was significantly inhibited, as detected by the MTT assay. (D and E) Downregulated UBAP2L induced cell cycle arrest at S phase in A172 cells. Scale bar, $100 \mu \mathrm{m} .{ }^{* * *} \mathrm{P}<0.001$. 

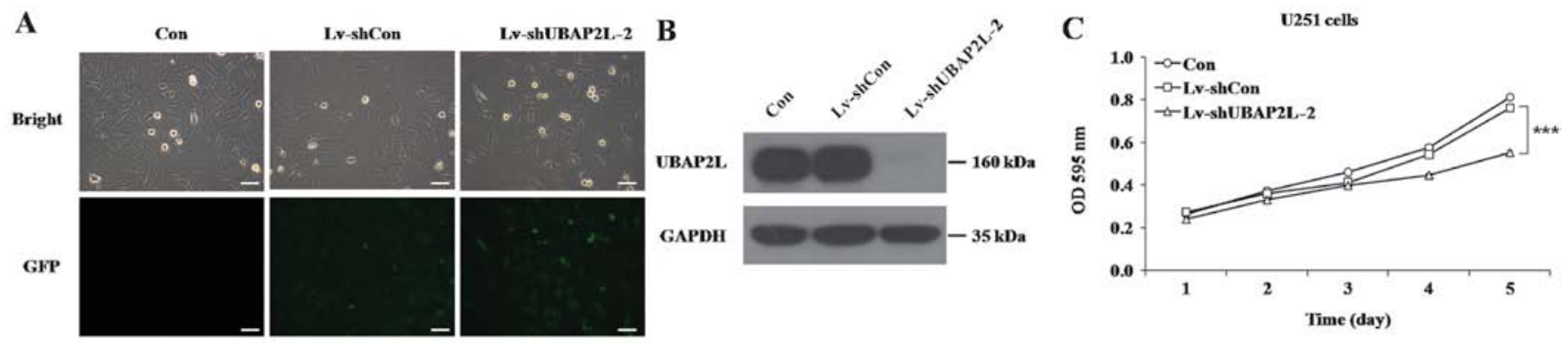

Figure 6. Effect of another Lv-shUBAP2L (Lv-shUBAP2L-2) on the proliferation of U251 cells. (A) Representative images of U251 cells after 4 days of lentivirus infection are shown. (B) Western blot analysis of ubiquitin-associated protein 2-like (UBAP2L) protein levels in U251 cells. The GAPDH protein was the internal control. (C) Cell proliferation in Lv-shUBAP2L-2 groups was significantly inhibited, as detected by the MTT assay. Con, cells without infection; Lv-shCon, cells infected with the Lv-shCon; Lv-shUBAP2L-2, cells infected with another Lv-shUBAP2L. ${ }^{* * *} \mathrm{P}<0.001$.

infection for 4 days (Fig. 5A). RT-qPCR showed that UBAP2L expression was reduced by $73.8 \%$ in A172 cells (Fig. 5B). The proliferation rate of A172 cells was also markedly decreased in the Lv-shUBAP2L group (Fig. 5C). In addition, the cell cycle distribution of A172 cells was examined following lentivirus infection (Fig. 5D). A clear increase of cell percentage in the $\mathrm{S}$ phase was observed in A172 cells (Lv-shUBAP2L, $41.41 \pm 0.24 \%$ vs. Lv-shCon, 26.04 $\pm 0.41 \%$; Fig. 5E), which could be due to the specific cell type.

Evaluation of lentivirus-mediated shRNA-targeting UBAP $2 L$ specificity. To exclude the off-target effect of shRNA, another shRNA against UBAP2L was constructed and transduced into U251 cells. Similarly, $>90 \%$ of U251 cells expressed GFP following infection with another Lv-shUBAP2L (Lv-shUBAP2L-2) (Fig. 6A), suggesting high-efficiency and high-specificity infection by the recombinant lentivirus. In addition, the level of endogenous UBAP2L expression was evidently downregulated in U251 cells infected with Lv-shUBAP2L-2 (Fig. 6B). Cell proliferation was also significantly impeded in response to Lv-shUBAP2L-2 infection $(\mathrm{P}<0.001$; Fig. 6C).

\section{Discussion}

Previous studies have demonstrated that UBAP2L is involved in the pathogenesis of several human malignant tumors. The study by Sawyer (9) identified UBAP2L as a candidate gene that showed amplification expression in myeloma. A recent study (10) based on correlating expression arrays and array comparative genomic hybridization $(\mathrm{CGH})$ data also found that UBAP2L showed amplification expression in $\mathrm{HCC}$, which is one of the early genomic events associated with HCC development. Naz and Dhandapani (11) reported that UBAP2L showed $97 \%$ homology at the nucleotide and amino acid sequences with ZPC-interacting protein involved in malignant ovarian tumors (15). Furthermore, Sudhir et al (16) identified UBAP2L as a novel target of mitogen-activated protein kinase (MAPK) family kinases that acts as a downstream component of Ras-mediated signaling and has an important role in the pathogenesis of certain types of human cancer, such as lung cancer and glioma (17). However, whether UBAP2L has a role in the tumorigenesis and progression of glioma via regulation of the MAPK pathway, has not yet been investigated.
In the present study, UBAP2L was ubiquitously expressed in five human glioma cell lines. To explore the function of UBAP2L in glioma, a loss-of-function analysis was performed via an shRNA-expressing lentivirus system, which is a safe non-toxic shRNA delivery method that ensures a long-lasting stable silencing effect $(18,19)$. U251 and U373 cells infected with Lv-shUBAP2L exhibited significant reductions in cell proliferation and colony formation, and an increase of cell population in the $\mathrm{G}_{0} / \mathrm{G}_{1}$ phase. Knockdown of UBAP2L in A172 cells also inhibited cell proliferation along with $S$ phase arrest, which showed a different regulatory mechanism of cell growth inhibition in specific glioma cell type.

In cancer cells, cell cycle is a critical mechanism of development, progression and resistance to treatment (2). Aberrant function of cell cycle regulators generally alters the properties of growth, differentiation and apoptosis in cancer cells (20). Previous studies have shown that the involvement of UPS in cell cycle regulation of glioma cells is critical. Piva et al (21) found that the cyclin-dependent kinase inhibitor (p27) was degraded in a proteasome-dependent manner, which provides evidence indicative of an association between the stability of cell cycle proteins and UPS in gliomas. Pamarthy et al (22) further showed that S-phase kinase-associated protein 2 (Skp2), which belongs to the Ub ligase F-box family, could promote $\mathrm{G}_{1}-\mathrm{S}$ transition through targeting of $\mathrm{p} 27$ for degradation. Amador et al (23) demonstrated that the $\mathrm{Ub}$ ligase APC/C (Cdc20) contributed to activation of CDK1 in early $\mathrm{M}$ phase in gliomas by controlling the UPS-dependent degradation of cell cycle-related protein (p21). An et al (24) suggested that UPS exerted an indirect role in the cell cycle of glioma cells by regulation of the oncoprotein c-Myc stability, which is known as an activator of cell cycle acceleration and involved in the $G_{1}$ phase. In addition, Rb and p53, which have crucial roles in cell cycle regulation, could be regulated by UPS $(25,26)$. In the present study, UBAP2L, which contains a UBA involved in UPS, was found to facilitate cell growth by regulating $G_{0} / G_{1}$ to $S$ phase progression in glioma. Therefore, previous studies that focus on the function and mechanism of UPS can provide a foundation for further studies regarding UBAP2L in malignant glioma.

In conclusion, the present study indicates that UBAP2L has a key role in glioma cell growth, suggesting that UBAP2L may act as an oncogene to promote the development of glioma via cell proliferation and cell cycle regulation. Further investigation 
is required to elucidate the precise molecular mechanisms by which UBAP2L affects human glioma progression.

\section{Acknowledgements}

The present study was supported by the National Natural Science Foundation of China (grant no. 81072066).

\section{References}

1. Wen PY and Kesari S: Malignant gliomas in adults. N Engl J Med 359: 492-507, 2008.

2. Hanahan D and Weinberg RA: Hallmarks of cancer: The next generation. Cell 144: 646-674, 2011.

3. Sakamoto KM, Kim KB, Verma R, Ransick A, Stein B, Crews CM and Deshaies RJ: Development of Protacs to target cancer-promoting proteins for ubiquitination and degradation. Mol Cell Proteomics 2: 1350-1358, 2003.

4. Wei $\mathrm{W}$ and Lin H-K: The key role of ubiquitination and sumoylation in signaling and cancer: A research topic. Front Oncol 2: 187, 2012.

5. Yamaguchi H, Hsu JL and Hung MC: Regulation of ubiquitination-mediated protein degradation by survival kinases in cancer. Front Oncol 2: 15, 2012.

6. Vlachostergios PJ, Voutsadakis IA and Papandreou CN: The ubiquitin-proteasome system in glioma cell cycle control. Cell Div 7: 18, 2012.

7. Ciechanover A: Intracellular protein degradation: From a vague idea through the lysosome and the ubiquitin-proteasome system and onto human diseases and drug targeting. Bioorg Med Chem 21: 3400-3410, 2013.

8. Wilde IB, Brack M, Winget JM and Mayor T: Proteomic characterization of aggregating proteins after the inhibition of the ubiquitin proteasome system. J Proteome Res 10: 1062-1072, 2011.

9. Sawyer JR: The prognostic significance of cytogenetics and molecular profiling in multiple myeloma. Cancer Genet 204 3-12, 2011 .

10. Zhu Z-Z, Wang D, Cong W-M, Jiang H, Yu Y, Wen BJ, Dong H, Zhang X, Liu SF, Wang AZ, et al: Sex-related differences in DNA copy number alterations in hepatitis B virus-associated hepatocellular carcinoma. Asian Pac J Cancer Prev 13: 225-229, 2012.

11. Naz RK and Dhandapani L: Identification of human sperm proteins that interact with human zona pellucida3 (ZP3) using yeast two-hybrid system. J Reprod Immunol 84: 24-31, 2010.
12. Madura K: The ubiquitin-associated (UBA) domain: On the path from prudence to prurience. Cell Cycle 1: 235-244, 2002.

13. Sakoda T, Kasahara N, Hamamori Y and Kedes L: A high-titer lentiviral production system mediates efficient transduction of differentiated cells including beating cardiac myocytes. J Mol Cell Cardiol 31: 2037-2047, 1999.

14. Livak KJ and Schmittgen TD: Analysis of relative gene expression data using real-time quantitative PCR and the 2(-Delta Delta C(T)) method. Methods 25: 402-408, 2001.

15. Rahman NA, Bennink HJTC, Chrusciel M, Sharp V, Zimmerman Y, Dina R, Li X, Ellonen A, Rivero-Müller A, Dilworth $S$, et al: A novel treatment strategy for ovarian cancer based on immunization against zona pellucida protein (ZP) 3 . FASEB J 26: 324-333, 2012.

16. Sudhir PR, Hsu CL, Wang MJ, Wang YT, Chen YJ, Sung TY, Hsu WL, Yang UC and Chen JY: Phosphoproteomics identifies oncogenic Ras signaling targets and their involvement in lung adenocarcinomas. PLoS One 6: e20199, 2011.

17. Olsen BB, Svenstrup TH and Guerra B: Downregulation of protein kinase CK2 induces autophagic cell death through modulation of the mTOR and MAPK signaling pathways in human glioblastoma cells. Int J Oncol 41: 1967-1976, 2012.

18. Bank A, Dorazio R and Leboulch P: A phase I/II clinical trial of beta-globin gene therapy for beta-thalassemia. Ann N Y Acad Sci 1054: 308-316, 2005.

19. Fang J, Yu Z, Lian M, Ma H, Tai J, Zhang L and Han D: Knockdown of zinc finger protein, X-linked (ZFX) inhibits cell proliferation and induces apoptosis in human laryngeal squamous cell carcinoma. Mol Cell Biochem 360: 301-307, 2012.

20. Sherr CJ: The Pezcoller lecture: Cancer cell cycles revisited. Cancer Res 60: 3689-3695, 2000.

21. Piva R, Cancelli I, Cavalla P, Bortolotto S, Dominguez J, Draetta GF and Schiffer D: Proteasome-dependent degradation of p27/kip1 in gliomas. J Neuropathol Exp Neurol 58: 691-696, 1999.

22. Pamarthy D, Tan M, Wu M, Chen J, Yang D, Wang S, Zhang H and Sun Y: p27 degradation by an ellipticinium series of compound via ubiquitin-proteasome pathway. Cancer Biol Ther 6: 360-366, 2007.

23. Amador V, Ge S, Santamaría PG, Guardavaccaro D and Pagano M: APC/C(Cdc20) controls the ubiquitin-mediated degradation of p21 in prometaphase. Mol Cell 27: 462-473, 2007.

24. An J, Yang DY, Xu QZ, Zhang SM, Huo YY, Shang ZF, Wang Y, Wu DC and Zhou PK: DNA-dependent protein kinase catalytic subunit modulates the stability of c-Myc oncoprotein. Mol Cancer 7: 32, 2008.

25. Ying $\mathrm{H}$ and $\mathrm{Xiao} Z \mathrm{ZX}$ : Targeting retinoblastoma protein for degradation by proteasomes. Cell Cycle 5: 506-508, 2006.

26. Ohgaki $\mathrm{H}$ and Kleihues P: Genetic pathways to primary and secondary glioblastoma. Am J Pathol 170: 1445-1453, 2007. 